\title{
Increased effort affects performance in a joint pick-and-place task
}

\author{
Tamara Lorenz, Anna Schubö
}

\begin{abstract}
This study examines how human movements are adapted when interacting with another person. It is also considered that the sequence of the movement can be to the choice of both actors. Different parameters of pick-and-place movements are compared in situations in which one person is moving alone or conjointly with a partner while the sequence of the task was predefined or made up by the participant. A schematic framework for the interpretation of increased effort is provided. Results are discussed in relation to improvements for human-robot-interaction.
\end{abstract}

\section{INTRODUCTION}

$\mathrm{W}$ hen thinking about the future - its technological abilities and comfort - we almost always picture robots that help us with challenges of our daily life. If that is industrial manufacturing or simply helping us in the household - in our predictions we interact very naturally with our artificial friends. Thus, in a human centered environment useful robots should carry out tasks or functions in a way that is socially acceptable, comfortable and effective for people they interact with [1]. For such an intuitive interaction between humans and robots it is necessary that unconscious signals sent by one interaction partner are perceived and understood by the other in the right way. With contemporary robots and assistive systems this intuitiveness is often impeded because robots do not move in a humanlike way and therefore it is more difficult for humans to anticipate the exact timing and spatial distribution of the robot's movements [2].

\section{A. From human-human interaction to human-robot interaction}

Research of cognitive psychologists and social neuroscientists attempts to explore the human ability to interact with other people by anticipating the future actions of their counterpart [3]. Different experiments show that kinematics of hand and arm movements are modulated due to the type of social context [4] and are affected by movements of other people acting in our close surrounding [5]. To achieve an intuitive interaction between humans and robots it is thus important to consider these modifications for the

Manuscript received March 11st, 2010. This work was funded by the German Research Foundation (Deutsche Forschungsgemeinschaft, DFG) as part of the Excellence Cluster "Cognition for Technical Systems" (CoTeSys, Project \#410). The authors like to thank Imogen Willers for her assistance in data collection.

Tamara Lorenz (+49-(0)89-289-25735; tamara.lorenz@psy.lmu.de) and Anna Schubö (anna.schuboe@lmu.de) are both with the Psychology Department (Experimental Psychology Unit), Ludwig-Maximilians University, Munich, Germany. development of robots. One approach to improve human robot interaction could be, to examine kinematic data from human-human interaction in order to better understand how people work together and in what way adaptive artificial systems need to act to provide a better predictability for human users [6].

\section{B. Movement planning}

If people want to work together successfully they have to agree on the outcome of their joint action. To achieve a shared goal, both cooperation partners have to plan their respective parts of an action in order to achieve the intended outcome [8]. Additionally it is important that these plans can be adapted to sudden changes. For example when somebody wants to hand over a pen and it falls down, our reaction is to try to catch it as fast as possible. Another situation could be that two people reach out for the same item. To avoid collisions at least one of them has to adapt his trajectory to prevent a collision. Obhi et al. [7] showed that switching from an internally generated action plan to an externally triggered mode of action production leads to increased reaction times. But it remains unclear whether the plan of how to achieve the action goal has an impact on movement kinematics.

Therefore we designed an experiment in which participants were given multiple possibilities to reach a certain end state. This provided us with the opportunity to see if movement parameters and reaction time are affected by free movement planning in comparison to a trained predefined action plan.

\section{Experimental design}

The main idea of the experiment is to explore how movement parameters of one person are affected when that person has to coordinate her actions with a partner. Vesper et al. [9] showed that movement coordination influences movement parameters and we hypothesize that it may even increase the effort of action planning. In general we think, that if the complexity of an action situation is enhanced, one should observe longer movement times with decreased velocities compared to the situation in which the same person performs the task with less effort (see Fig. 1). Therefore a task was designed, in which wooden building bricks had to be picked up and transported to a specific target position. Each person had to care for four bricks. During interaction people were told to move the bricks alternately. Thus, adding another person to the action situation would increase task complexity for the acting person. One can assume that in such an interaction situation, longer trajectories for the transportation movements may be 


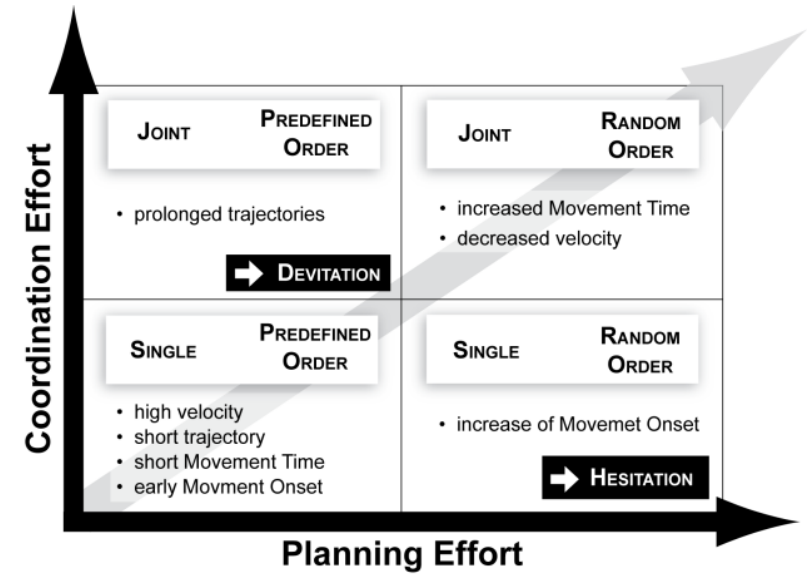

Fig. 1. Background for experimental design. When task complexity increases (grey arrow) either due to higher planning effort (x-axis) or due to additional coordination effort (y-axis) then velocity should decrease and movement time should become longer (upper right corner). Thus if the effort is increased in only one direction, also increased Movement Time and decreased velocity should be found. In addition, if only the planning effort is increased the Movement onset should be longer due to hesitations in reaction time. On the other side if only coordination effort is increased longer trajectories due to deviations should be observed.

observed that are caused by deviations due to movement coordination and collision avoidance [9]. Another way to increase task complexity may be to include a planning component by allowing participants to perform the action in an individually selected, random order. We hypothesize that this will increase the planning effort and therefore cause a delay in movement onset time. Although movement times are expected to be longer, the path length should not be affected. We expect people to rather hesitate in a planning period than move around without a completed movement plan. Summing up one would expect longer movement times and lower velocities for both increased planning and coordination effort tasks. If only coordination effort is enhanced, one may find elongated trajectories which are not necessarily expected for prolonged planning periods. Thus, longest movement times, lowest velocities and longest trajectories are expected in a situation in which participants perform together with a partner while at the same time choosing their own sequence order.

\section{METHOD}

\section{A. Participants}

Twelve pairs of participants ( 9 men, 15 women, age: 21 to 30 years, mean 24.46 years) were tested in the experiment. They were students of local universities. All were right handed and had normal or corrected to normal vision. For participation they were paid 8 Euros per hour.

\section{B. Apparatus, material and experimental setup}

For each trial eight wooden building bricks of the size $4 \mathrm{x}$ $4 \mathrm{x} 4 \mathrm{~cm}$ were used. In order to distinguish them, they were marked in different colors. The bricks were placed in front of the participants on starting positions that were marked on the table (see Fig. 2). The instructed task was to pick up the bricks from their initial position and place them at the respective target position so that each brick had to be moved the same distance of $30 \mathrm{~cm}$. During task performance, participants' hand movements were recorded with a Polhemus Liberty 240/8 system, a magnetic motion tracker with six degrees of freedom (X, Y and $\mathrm{Z}$ coordinates and the three rotation angles) and a constant sampling rate of 240 $\mathrm{Hz}$. Three sensors were placed centrally on the back of the thumb, index finger and middle finger of each participant's right hand. For being able to review task performance later, all movements were recorded with a video camera. Experimental procedure, data collection and preparation for statistical analyses were done with Matlab 2006b. Statistical analyses were performed with PASW (SPSS).

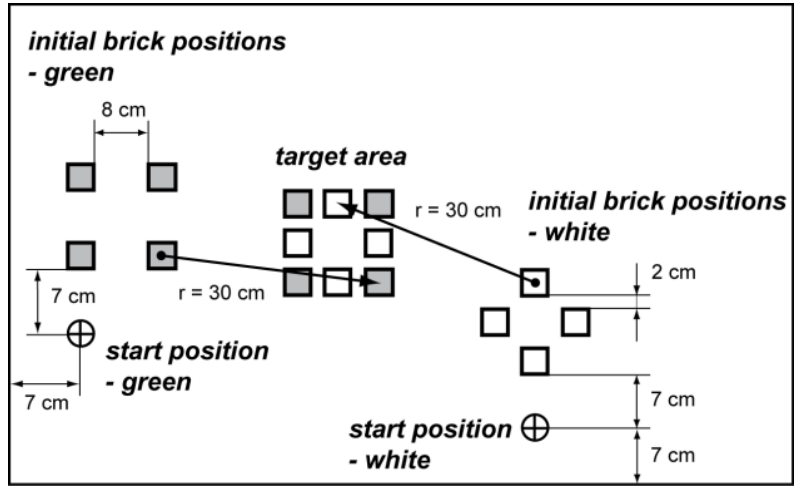

Fig. 2. Experimental Setup. Participants were sitting on a table in a cater-cornered arrangement. Instructions were presented on a computer screen which was well-visible for both participants. The left-sitting person had to move four green bricks, the right sitting person four white bricks. For both colors an initial area was defined in which the bricks were positioned before the start of each trial. Only the data of the right sitting person's right hand was analyzed.

\section{Task and procedure}

Participants had to perform a sequence of pick-and-place movements with their right hands. The task was to pick up building bricks from an initial storage area and place them at individually corresponding target positions. Before the experiment started, participants were instructed verbally and in written form. As the seating position was crucial for the experiment it was assigned indiscriminately. Until the end of the experiment, participants were kept naïve with respect to the research goal. Eight single and eight joint experimental blocks were performed; the blocks were alternated and the order was balanced across participants. Each block consisted of eight trials. In the single condition the right sitting person was performing the whole block alone by moving the white bricks with the right hand while the left sitting person remained at the table with the hands placed off the table. In the joint condition the interaction partners had to cooperate in building the brick pattern in the target area by alternately moving their individual bricks also with the right hand. For each level of social context (single vs. joint) two sequence 


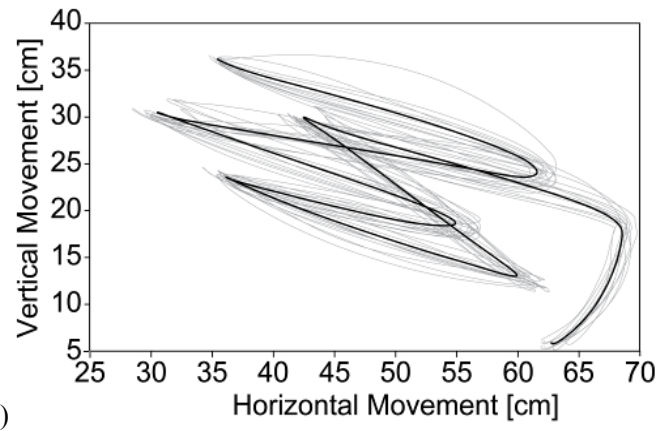

(a)

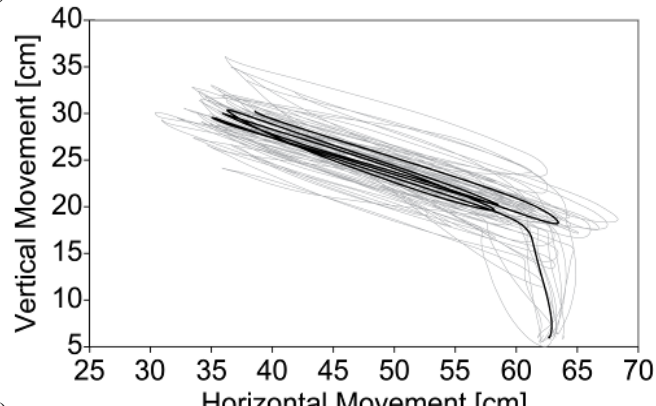

(b)

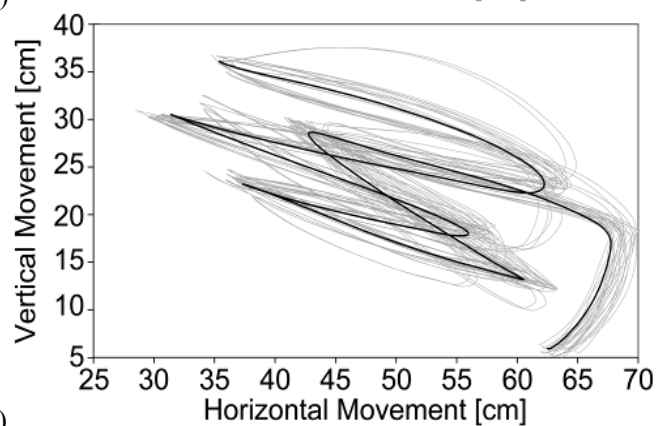

(c)

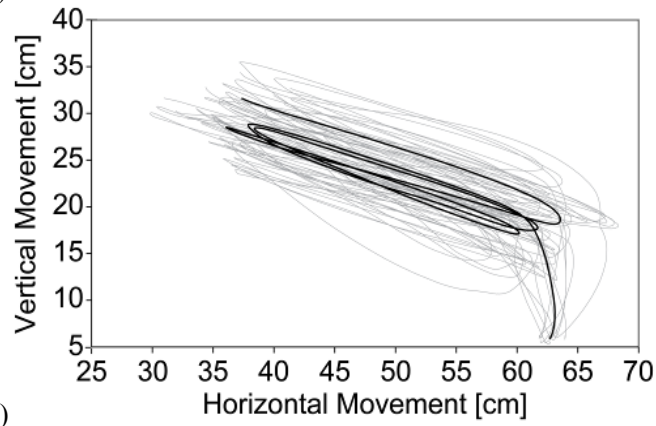

Fig. 3. Average trajectories for (a) single predefined, (b) single random, (c) joint predefined, and (d) joint random condition. Grey lines average per participant, the bold line shows the average across all participants. From this average it can be seen that in random sequence order trials participants performed the trajectories not in a predefined but in a self-chosen, random order. Additionally a slight increase in deviations is visible for the joint condition $(c, d)$.

levels were introduced. Participants were instructed to either move their bricks in a predefined order that was presented on the screen before the trial or to choose a random order by themselves. In each experimental block each combination was presented four times and in completely randomized order. In total 16 blocks were performed with eight trials each. This led to a total of $16 * 8=128$ trials with $16 * 2=$ 32 repetitions for each experimental condition. At the beginning of the experiment participants performed two joint and two single practice blocks. Another practice block was included before both the first single and the first joint experimental block. Before each trial, participants had to place the index finger and thumb of the respective hand(s) on a starting cross marked on the table. The experimenter then pressed the control button that triggered the instruction presented on the screen in which starting order of the participants and the order of sequence for brick placement were specified. After three seconds the screen turned black and an acoustical start signal was presented. Simultaneously with the start signal the motion measurement was started. Participants now had to pick up the bricks and place them on the respective target area while following the instructed (predefined order condition) or their own sequence (random order condition). After the last brick was being placed they moved their hands back to the starting position. When they were done, the experimenter stopped data recording by pressing the control button again. The bricks were moved back to their initial positions and participants were instructed to put their hands back to the marked starting position so that the experimenter could start the next trial.

\section{DATA ANALYSIS}

Data Analysis focused on the right-hand movements of the right-sitting person. These movements allowed a direct comparison of the influence of the different social contexts and sequence conditions. Various parameters were computed in order to analyze the complete movement phase as well as several movement intervals. First, the error rate was calculated by classifying the error trials (noted by the experimenter and/or via video analysis). Trials in which bricks fell down, the brick sequence was performed in the wrong order, bricks were placed incorrectly at the goal position or participants started before the acoustical signal were marked as errors regardless of whether the left- or right-sitting person was responsible for them. Only valid trials were used for further statistical analyses. Velocity was calculated from the Euclidian distance between data points in the $\mathrm{X}, \mathrm{Y}$ and $\mathrm{Z}$ coordinates. A fourth order low-pass Butterworth filter with a cut-off frequency of $10 \mathrm{~Hz}$ was applied. The resulting phase shift was corrected by applying the same filter reversely. Movement onset was defined as the time participants needed to start their active movement after the start signal was presented. This time point was defined as the last minimum in the velocity profile before the peak that indicated the first reach-to-grasp movement. Additionally the velocity threshold for the movement onset was set to $20 \mathrm{~cm} / \mathrm{s}$ to exclude smaller movements that appear during the planning phase or while observing the partner. For each trial, the movement trajectory of the right-sitting person's right hand was explored on the basis of the velocity profile. The overall movement phase began with the movement onset (see Fig. 4) and ended with the placement of the last brick. 


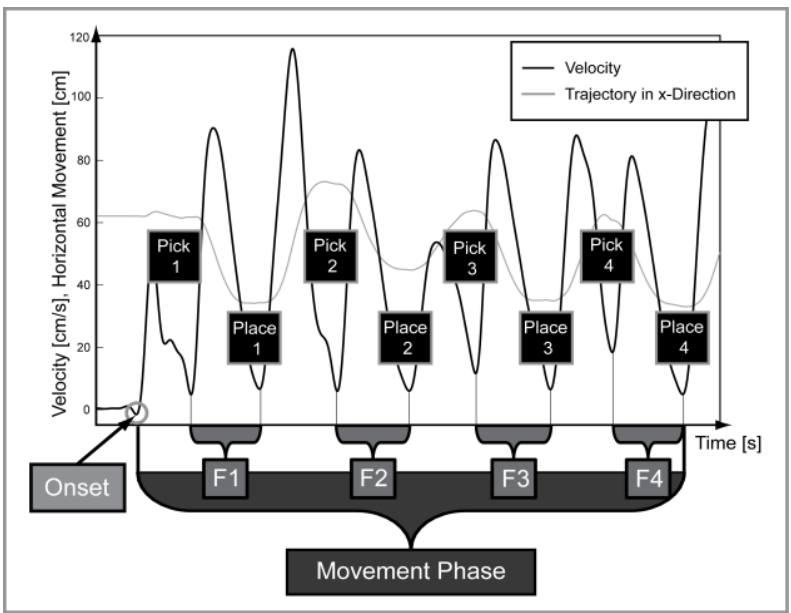

Fig. 4. Segmentation of the overall movement phase (example data of one trial). When a minimum in the velocity profile approaches zero, the movement is about to stop and either a picking or placing movement takes place. The overall movement phase starts with the onset of the Movement and ends with the placement of the fourth brick.

\section{A. Pick-and-place profile}

The overall movement phase was segmented in order to get detailed information about different parts of the movement. Whenever velocity approached zero, the movement of the arm was considered to be about to stop and either a picking or placing movement was conducted.

After the onset, the first peak in the velocity profile was seen as part of the movement that was conducted to reach out for the first brick. This part was followed by the first of four forwards movements (F1-F4) which consisted of picking and placing the respective brick. The underlying direct path, the 2D-distance between initial position of one brick and its corresponding target position, was always equal for the forwards movements (compare also Fig.2). Between two forwards movements participants had to move their hand back to the initial area to reach for the next brick.

\section{Results}

Data was analyzed using a $2 \times 2$ repeated measures analysis of variance (ANOVA) with the within-subject factors social context (single vs. joint) and sequence order (predefined vs. random).

\section{A. Error rate}

In total $7.62 \%$ of all trials were excluded due to errors. No differences were found between single and joint performance $\left(\mathrm{M}_{\mathrm{S}}=2.63 \%, \mathrm{M}_{\mathrm{J}}=2.25 \%, \mathrm{SEM}>.30 \%\right)$ or between predefined and random sequence trials $\left(\mathrm{M}_{\mathrm{P}}=2.46 \%, \mathrm{M}_{\mathrm{R}}=\right.$ $2.42 \%$, SEM $>0.5 \%$ ). Also no interaction effects were found between factor levels.

\section{B. Overall movement phase}

For the overall movement phase all comparisons reached significance in the parameters movement time, path length and mean velocity. More precise, when people had to perform the movements alone they needed less time compared to when working conjointly with a partner $\left(\mathrm{M}_{\mathrm{S}}=\right.$ $5.98 \mathrm{~s}, \mathrm{M}_{\mathrm{J}}=7.03 \mathrm{~s}, \mathrm{SEM}_{\mathrm{S}}=0.38 \mathrm{~s}, \mathrm{SEM}_{\mathrm{J}}=0.44 \mathrm{~s}, F(1,11)$ $=21.99, p=0.001)$. They also passed shorter ways in single than in joint trials $\left(\mathrm{M}_{\mathrm{S}}=289.76 \mathrm{~cm}, \mathrm{M}_{\mathrm{J}}=306.65 \mathrm{~cm}, \mathrm{SEM}_{\mathrm{S}}\right.$ $\left.=3.01 \mathrm{~cm}, \mathrm{SEM}_{\mathrm{J}}=6.00 \mathrm{~cm}, F(1,11)=10.10, p<0.01\right)$. The mean velocity was higher for single than for joint performance $\left(\mathrm{M}_{\mathrm{S}}=48.69 \mathrm{~cm} / \mathrm{s}, \mathrm{M}_{\mathrm{J}}=41.73 \mathrm{~cm} / \mathrm{s}, \mathrm{SEM}_{\mathrm{S}}=\right.$ $\left.2.97 \mathrm{~cm} / \mathrm{s}, \mathrm{SEM}_{\mathrm{J}}=1.75 \mathrm{~cm} / \mathrm{s}, F(1,11)=19.19, p=0.001\right)$.

In trials in which participants had to plan the sequence order of their movements themselves they needed more time than when the sequence was predefined $\left(\mathrm{M}_{\mathrm{P}}=6.40 \mathrm{~s}, \mathrm{M}_{\mathrm{R}}=\right.$ $6.61 \mathrm{~s}, \mathrm{SEM}_{\mathrm{P}}=0.38 \mathrm{~s}, \mathrm{SEM}_{\mathrm{R}}=0.41 \mathrm{~s}, F(1,11)=19.14, p=$ 0.001). Also shorter trajectories were observed for predefined sequence order $\left(\mathrm{M}_{\mathrm{P}}=294.51 \mathrm{~cm}, \mathrm{M}_{\mathrm{R}}=301.90\right.$ $\mathrm{cm}, \mathrm{SEM}_{\mathrm{P}}=4.17 \mathrm{~cm}, \mathrm{SEM}_{\mathrm{R}}=3.87 \mathrm{~cm}, F(1,11)=34.121$, $p<0.001)$. Mean velocity was lower for random sequence order $\left(\mathrm{M}_{\mathrm{P}}=45.74 \mathrm{~cm} / \mathrm{s}, \mathrm{M}_{\mathrm{R}}=44.68 \mathrm{~cm} / \mathrm{s}, \mathrm{SEM}_{\mathrm{P}}=2.30\right.$ $\left.\mathrm{cm} / \mathrm{s}, \mathrm{SEM}_{\mathrm{R}}=2.32 \mathrm{~cm} / \mathrm{s}, F(1,11)=12.80, p<0.01\right)$. For the movement time and the path length also significant interaction effects were observed (MT: $F(1,11)=6.44, p<$ 0.05; PL: $F(1,11)=21.07, p=0.001)$. Results for the overall movement phase are shown in Fig. 5.

\section{Movement onset}

When comparing the movement onset (see Fig. 6) for single and joint tasks, only numerical differences were found
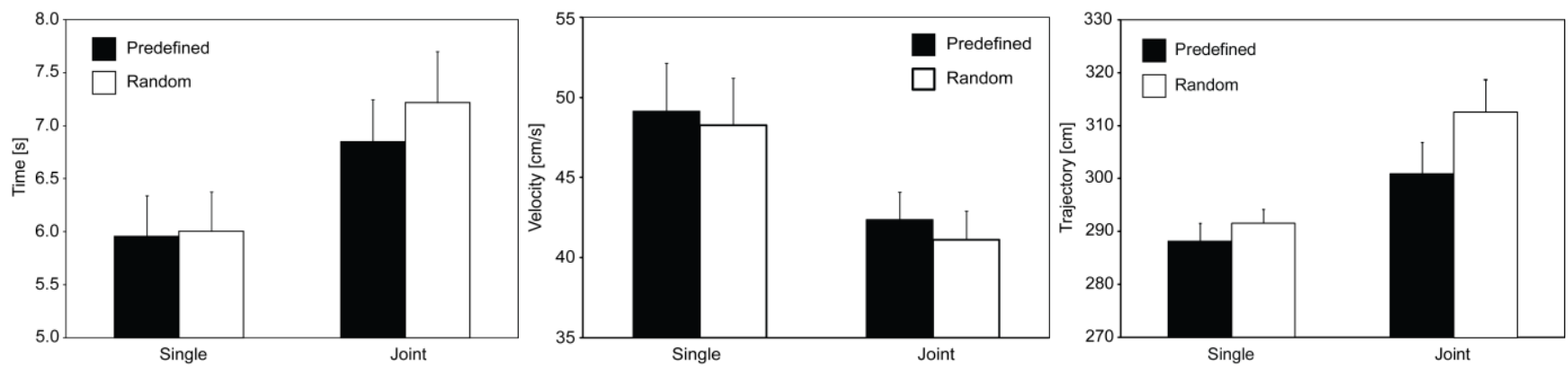

Fig. 5. Results for the overall movement phase. Left panel shows the movement time, intermediate panel shows mean velocity and the right panel shows the path length. Results are shown separately for single (left columns) and joint (right columns) performance and for predefined (filled bars) and random (unfilled bars) sequence order. 


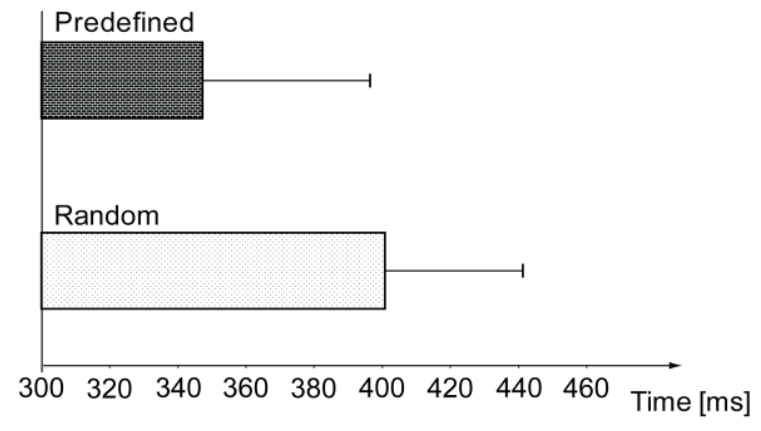

Fig. 6. Movement onset. In the random sequence order task (lighter bar) Movement onset was increased compared to the Movement onset in the predefined sequence order task (darker bar).

that did not reach significance $(p>0.1)$. But movement onset was higher for random than for predefined sequence order $\left(\mathrm{M}_{\mathrm{P}}=347 \mathrm{~ms}, \mathrm{M}_{\mathrm{R}}=401 \mathrm{~ms}, \mathrm{SEM}_{\mathrm{P}}=49 \mathrm{~ms}, \mathrm{SEM}_{\mathrm{R}}=40 \mathrm{~ms}\right.$, $\mathrm{F}(1,11)=4.81, \mathrm{p}=0.05)$.

\section{DISCUSSION}

This study aimed at exploring human behavior in a pickand-place task. In a basic setting one person (single) had to pick up four building bricks in a given sequence order (predefined) and move them to a defined target position. Task complexity was increased in two ways: first another person was added (joint) to create an interaction situation and thereby enhance the level of coordination effort; second, to achieve additional planning effort, the sequence order of the task was left up to the participant (random).

\section{A. Effects of joint action}

Concerning the interaction situation this study showed that people needed more time when they worked together with a partner. They also passed longer trajectories with a lower velocity when they had to coordinate their movements with somebody else. Thus, when people worked alone, they were faster, moved with higher velocity and passed shorter ways to complete the task.

In addition to the effects in the real interaction situation it is also interesting to explore how the knowledge about different social context situations (single /joint) affects the time before the movement is first started [3]. But for the movement onset no significant difference between single and joint performance was found. This provides support to the findings of Vesper et al. [9] who suggested that people apply a strategic adaptation of own movement parameters only in the action situation itself. In specific this means that the same amount of time is needed to process the visual stimuli and plan the movement before starting a single or a joint task.

\section{B. Effects for random sequence order}

Apart from creating an interaction situation, complexity of a task can also be enhanced by leaving the sequence order of the bricks up to the participant (random sequence order). In that case, participants needed more time to perform the overall movement phase. In the same time period they furthermore passed longer trajectories with a lower mean velocity.

To see if the planning effort is also affected by introducing a random sequence order task, the movement onset was analyzed. And in fact, the time to actually start the movement was longer for random compared to predefined sequence order.

\section{Interaction effects}

Statistical Data Analysis was performed using an analysis of variances in order to compare the within-subject factors social context (single vs. joint) and sequence order (predefined vs. random). This method allowed to also have a look on interaction effects between factors. For the overall movement phase, significant interaction effects between social context and sequence order were found for the movement time and the path length. When comparing the results with the graphs in Fig. 5 one can assume that the differences between single and joint were stable. This means that movement time in the joint predefined condition was longer than movement time in the single predefined condition and joint random took longer than single random.

In contrast, the differences between predefined and random sequence order mainly appeared for the joint action case. More precise, in the overall movement phase movement time for the joint random condition was longer than for the joint predefined condition whereas no difference appears between movement times in single random and single predefined performance. The same pattern also applied for the path length.

\section{Increased effort}

In line with the predictions (see Fig.1) people needed more time as soon as the effort was increased in either way. Simultaneously velocity decreased. More specific, shortest movement times with highest velocities were examined in the single predefined condition and longest times with lowest velocities in the joint random task. The results show that if complexity of a task is increased through adding another person (joint) or providing additional planning aspects (random), movement parameters were affected and it seems that the effort to finish the task was increased. In more detail, when participants were asked to work conjointly they not only needed more time and slowed down, they also passed longer ways. Vesper et al. [9] claimed that people most likely move furthest away from the direct path in a joint condition to avoid nearing the partner and such avoid collisions. This indicates that more deviations were executed in a joint task. In summary, higher movement times, slower velocities and prolonged trajectories were found for the overall movement phase in a joint situation compared to single performance. Thus, results support the idea that adding another person leads to increased coordination effort as suggested in Fig.1 (upper panels).

When looking on the effects of sequence order for the overall movement phase, prolonged trajectories were observed in random sequence trials. Also a significant interaction effect was observed in this situation: When 
looking on Fig. 5a (right panel) one can see that the difference in path length between predefined and random sequence order mainly appeared in the joint condition (compare also section V.C). Considering this, one could assume that the effort to coordinate one's own movements was even harder when people could only guess or anticipate the movement sequence of the counterpart in the joint random case. Thus, when people had to coordinate their movements with somebody who's next movements were not instructed (and therefore not known) and in the same time had to select their own brick sequence order, they might have produced even longer trajectories than in a joint predefined task due to a strategic path adaptation. This strategy would include bigger deviations that were already included into the action plan because of the knowledge that the interaction partner might behave unexpectedly. The fact that for the overall movement phase no differences were found between single random and single predefined movements supports this idea because in single performance no online adaptation to a partner is necessary. An additional explanation for the non-existing path length difference between single predefined and single random performance during the overall movement phase could be, that people, when they work alone, already create an action and sequence plan before the movement even started. Further evidence for that theory comes from findings in the movement onset. People needed more time to start - and plan - a random sequence order than a predefined one.

As a conclusion it can be said, that the scheme that was introduced in the beginning provides a good way to understand how movement parameters are affected by increasing the complexity of a task either through adding another person to enhance coordination effort or through leaving the sequence order of the task up to the participant to increase planning effort.

\section{ADVANTAGES FOR HUMAN-ROBOT INTERACTION}

Current robots research often draws attention to neuroscientific questions. Erlhagen and Bicho [10] claimed that to efficiently interact with another agent in solving a mutual task, a robot should be endowed with cognitive skills such as action understanding and prediction amongst others. To develop a model for a joint search task they included knowledge from cognitive sciences and succeeded.

Following this line, the study at hand aimed to provide principles that can be used for human-robot interaction. The schematic framework (Fig. 1) gives a good guideline on how humans adapt their behavior when the task effort is increased. Thus, when an interaction between a human and a robot takes place, the robot can use this information to better adapt his own behavior to the parameters of the human movements. For example, to establish a natural movement, in the robotic arm-trajectory, the deviation from the direct path should be larger than in a movement that the robot is performing alone while only being observed by a human actor - even if the observation is for adaptation processes.

Another problem, developers face while thinking of human robot interaction is the definition of task sequence. If an interaction between humans and robots is required, these days the order of items to be used is usually hardcoded. But if it comes to a situation in which humans and robots should for example interact in a domestic environment it can be unimportant in which order the items are removed from the table. Therefore it is useful for a robot to know that also for the human cognitive processes for item selection are involved. Additionally, the movement velocity goes down when the order of items is random. This is an important factor for prediction of the other's position in relation to the own one and thus for collision avoidance.

In general it is always important to accomplish effective coordination of the human's and the robot's limbs. Therefore it is necessary that the robot can predict the human state and action plan. The provided framework is a good step into that direction and might even provide a guideline for further investigation of human behavior.

\section{REFERENCES}

[1] M.L. Walters, K.L. Koay, D.S. Syrdal, K. Dautenhahn, and R. Boekhorst, "Preferences and Perceptions of Robot Appearance and Embodiment in Human-Robot Interaction Trials.," New Frontiers in Human-Robot Interaction, Adaptive and Emergent Behaviour and Complex Systems (AISB09), K. Dautenhahn, Edinburgh, Scotland: SSAISB, 2009, pp. 136-143.

[2] D.M. Wolpert, K. Doya, and M. Kawato, "A unifying computational framework for motor control and social interaction," Philosophical transactions of the Royal Society of London. Series B, Biological sciences, vol. 358, 2003, pp. 593-602.

[3] N. Sebanz, H. Bekkering, and G. Knoblich, "Joint action: bodies and minds moving together," Trends in cognitive sciences, vol. 10, 2006, pp. 70-76.

[4] I. Georgiou, C. Becchio, S. Glover, and U. Castiello, "Different action patterns for cooperative and competitive behaviour," Cognition, vol. 102, 2007, pp. 415-433.

[5] M.J. Richardson, W.L. Campbell, and R.C. Schmidt, "Movement interference during action observation as emergent coordination," Neuroscience letters, vol. 449, 2009, pp. 117-22.

[6] C. Vesper, S. Stork, and A. Schubö, "Movement Times in Inter- and Intrapersonal Human Coordination," 2008 ECSIS Symposium on Learning and Adaptive Behaviors for Robotic Systems (LAB-RS), Edinburgh: IEEE, 2008, pp. 17-22.

[7] S.S. Obhi, S. Matkovich, and S.J. Gilbert, "Modification of planned actions," Experimental brain research, vol. 192, 2009, pp. 265-74.

[8] G. Knoblich and N. Sebanz, "Evolving intentions for social interaction: from entrainment to joint action," Philosophical transactions of the Royal Society of London. Series B, Biological sciences, vol. 363, 2008, pp. 2021-31.

[9] C. Vesper, A. Soutschek, and A. Schubö, "Motion coordination affects movement parameters in a joint pick-and-place task," Quarterly journal of experimental psychology, vol. 62, 2009, pp. 2418-32.

[10] W. Erlhagen and E. Bicho, "The dynamic neural field approach to cognitive robotics," Journal of neural engineering, vol. 3, 2006, pp. R36-54. 